\title{
Inactivation of Macrophage Rab7 by Burkholderia cenocepacia
}

\author{
Kassidy K. Huynh ${ }^{\mathrm{a}}$ Jonathan D. Plumb ${ }^{\mathrm{a}}$ Gregory P. Downey ${ }^{\mathrm{c}}$ \\ Miguel A. Valvano ${ }^{\text {b Sergio Grinstein }}{ }^{a}$

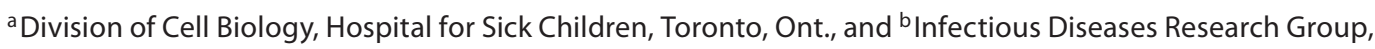 \\ Siebens-Drake Research Institute, Department of Microbiology and Immunology, University of Western Ontario, \\ London, Ont., Canada; ${ }^{C}$ Departments of Medicine and Pediatrics, National Jewish Health, Denver, Colo., and \\ Departments of Medicine and Integrated Department of Immunology, University of Colorado, Aurora, Colo., USA
}

\section{Key Words}

Burkholderia $\cdot$ Macrophage $\cdot$ Phagosome $\cdot$ Rab7 •

Cystic fibrosis

\begin{abstract}
Strains of the Burkholderia cepacia complex can survive within macrophages by arresting the maturation of phagocytic vacuoles. The bacteria preclude fusion of the phagosome with lysosomes by a process that is poorly understood. Using murine macrophages, we investigated the stage at which maturation is arrested and analyzed the underlying mechanism. Vacuoles containing B. cenocepacia strain J2315, an isolate of the transmissible ET12 clone, recruited Rab5 and synthesized phosphatidylinositol-3-phosphate, indicating progression to the early phagosomal stage. Despite the fact that the $B$. cenocepacia-containing vacuoles rarely fused with lysosomes, they could nevertheless acquire the late phagosomal markers CD63 and Rab7. Fluorescence recovery after photobleaching and use of a probe that detects Rab7-guanosine triphosphate indicated that activation of Rab7 was impaired by $B$. cenocepacia, accounting at least in part for the inability of the vacuole to merge with lysosomes. The Rab7 defect was not due to excessive cholesterol accumulation and was confined to the infected vacuoles. Jointly, these ex-
\end{abstract}

periments indicate that $B$. cenocepacia express virulence factors capable of interfering with Rab7 function and thereby with membrane traffic.

Copyright $\odot 2010$ S. Karger AG, Basel

\section{Introduction}

Cystic fibrosis (CF) is a debilitating genetic disease affecting multiple organs, in particular the lungs. Persons carrying mutations in the gene associated with CF have defective mucociliary clearance and impaired innate immunity of the airways [1-3]. Therefore, CF patients become highly susceptible to chronic respiratory infections with acute exacerbations by opportunistic pathogens, in particular Pseudomonas and Burkholderia species. Because these bacteria are notoriously resistant to most available antibiotics [4], chronic infection mediates progressive pulmonary deterioration leading to substantial morbidity and mortality, representing a serious health concern for CF patients. The Burkholderia cepacia complex comprises at least 17 closely related species $[5,6]$; from these, B. multivorans and B. cenocepacia are highly prevalent in CF patients [5].

\section{KARGER}

(C) 2010 S. Karger AG, Basel

Fax +41613061234

E-Mail karger@karger.ch

www.karger.com
Dr. Sergio Grinstein

Division of Cell Biology, Hospital for Sick Children

555 University Ave.

Toronto, ON M5G 1X8 (Canada)

Tel. +1 416813 5727, Fax +1 416813 5028, E-Mail sergio.grinstein@ sickkids.ca 
Table 1. Inventory of fluorescent probes and their targets

\begin{tabular}{llll}
\hline Plasmid & Construct & Target/organelle & Ref. \\
\hline pEGFP-Rab5a & GFP-Rab5 & early endosomes & 9 \\
P40PX-EGFP & GFP-PX & early endosomes/PI(3)P & 10 \\
EGFP-Rab7 wild type & GFP-Rab7 & late endosomes/lysosomes & 11,12 \\
EGFP-Rab7Q67L & GFP-Rab7(Q67L) & not applicable & 11,12 \\
RILP-C33-EGFP & RILP-C33-GFP & late endosomes/lysosomes, active Rab7 & 11,12 \\
pEGFP-CD63 & CD63-GFP & late endosomes/lysosomes & 13 \\
\hline
\end{tabular}

Innate immunity provides the first line of defense against infection. Leukocytes, primarily neutrophils and macrophages, contribute to the early stages of immunity, removing pathogens by an active process termed 'phagocytosis'. Phagocytosis is initiated upon engagement of receptors on the leukocyte surface, which signal reorganization of the actin cytoskeleton to extend pseudopods that encircle the pathogen, culminating in its internalization into a membrane-bound vacuole. Such phagocytic vacuoles, known as phagosomes, proceed to acquire microbicidal and degradative capabilities by sequential fusion with early endosomes, late endosomes and, lastly, lysosomes. These events, collectively termed 'phagosome maturation', render the phagosome lumen extremely acidic and rich in lytic enzymes, attributes that promote the elimination of engulfed pathogens.

Our previous research showed that B. cenocepacia J2315, a clinical isolate of the highly transmissible ET12 clone [7], was internalized by macrophages and targeted to a phagosome-like membrane-bound organelle, hereafter referred to as the $B$. cenocepacia-containing vacuole (BcCV).

However, unlike normal phagosomes, the $\mathrm{BcCV}$ failed to become acidic and, as a result, the bacteria remained viable for extended periods inside the macrophages [7]. Thus, it became apparent that B. cenocepacia could alter the course of phagosome maturation to its advantage, ensuring survival in the otherwise hostile environment of a phagosome. Bacterial survival and persistence in macrophages is believed to play a key role in the pathogenesis of B. cenocepacia [for a review, see ref. 8]. However, the means whereby $B$. cenocepacia co-opts maturation are not understood. To better understand the mechanism of $B$. cenocepacia intracellular infection at the molecular level, we analyzed the effect of strain J2315 on defined steps of the maturation sequence, using RAW 264.7 macrophages as a model system.

Inactivation of Macrophage Rab7 by Burkholderia

\section{Materials and Methods}

\section{Reagents and Plasmids}

Fetal bovine serum, Dulbecco's modified Eagle's medium (DMEM), Hepes-buffered RPMI-1640 (hereafter referred to as HPMI-1640) and phosphate-buffered saline (PBS) were from Wisent (St. Bruno, Que., Canada). Human serum was isolated from heparinized blood obtained by venipuncture of healthy donors and was filtered through $0.22-\mu \mathrm{m}$ Millipore Steriflip filters. Tetramethylrhodamine- and Alexa 647-conjugated dextran (MW 10,000) FM1-43 were purchased from Molecular Probes/Invitrogen. Luria Bertani (LB) broth was obtained from BioShop. Trimethoprim and N,N-dimethylacetamide were from Sigma. Arabinose was from Calbiochem and filipin was from Polysciences, Inc. Fugene HD transfection reagent was from Roche. The description, source and targets of the plasmids used are summarized in table 1. Construction of the plasmids encoding green fluorescent protein (GFP)-Rab5 and GFP-PX was described in detail elsewhere $[9,10]$. GFP-Rab7, Rab7(Q67L) and RILP-C33-GFP cDNA were kind gifts of Dr. Cecilia Bucci (Università degli Studi di Napoli Federico II, Naples, Italy) described previously [11, 12]. CD63 cDNA was the kind gift of Dr. G.M. Griffiths (William Dunn School of Pathology, Oxford, UK).

\section{Cell Culture and Transfection}

RAW264.7 macrophages (hereafter referred to as RAW cells/ macrophages) obtained from the American Type Culture Collection were grown in DMEM and 5\% heat-inactivated fetal bovine serum at $37^{\circ} \mathrm{C}$ under a $5 \% \mathrm{CO}_{2}$, humidified atmosphere. Transfections were performed using Fugene HD according the manufacturer's instructions.

\section{Bacterial Culture, Fixation and Opsonization}

B. cenocepacia J2315 (hereafter referred to as B. cenocepacia), a prototypic isolate of the transmissible ET12 clone, has been described previously $[14,15]$. Bacteria were grown at $37^{\circ} \mathrm{C}$ in $\mathrm{LB}$ medium with shaking. $B$. cenocepacia derivatives carrying pJR1 [monomeric red fluorescent protein (RFP)] or pMLBAD-enhanced GFP (eGFP) were grown in the presence of a final concentration of $100 \mu \mathrm{g} / \mathrm{ml}$ trimethoprim dissolved in N,N-dimethylacetamide. Also, 2\% (weight/vol) arabinose was added to induce expression of eGFP in B. cenocepacia containing pMLBAD-eGFP. Escherichia coli $\mathrm{DH} 5 \alpha$ expressing monomeric RFP were grown in LB medium with ampicillin. Bacteria were sedimented by cen- 
trifugation and washed with HPMI-1640 several times prior to infection. Aliquots containing 3-4 × $10^{8}$ bacterial cells were opsonized with $500 \mu \mathrm{l}$ of filtered human serum (diluted 1:4 in PBS) for $30 \mathrm{~min}$ at $37^{\circ} \mathrm{C}$ with agitation. Bacteria were washed several times with HPMI-1640 and homogenized lightly using a Dounce homogenizer to break up any clumps. Bacteria were either left untreated (hereafter referred to as 'live') or fixed at room temperature with $8 \%$ ( $\mathrm{vol} / \mathrm{vol}$ ) paraformaldehyde in PBS for $1 \mathrm{~h}$ (hereafter referred to as 'dead'), then quenched with $10 \mathrm{mM} \mathrm{NH}_{4} \mathrm{Cl}$, washed and resuspended in HPMI-1640.

\section{Phagocytosis Protocol}

RAW macrophages grown on glass coverslips (Fisher, Pittsburgh, Pa., USA) were bathed in serum-free DMEM prewarmed to $37^{\circ} \mathrm{C}$ and overlaid with either live or fixed bacteria at a multiplicity of infection (MOI) of 60 , followed by centrifugation at $300 \mathrm{~g}$ for $1 \mathrm{~min}$ to deposit the bacteria on the cell surface and thereby synchronize phagocytosis. Macrophages with bacteria were incubated for $20 \mathrm{~min}$ at $37^{\circ} \mathrm{C}$ in a humidified $5 \% \mathrm{CO}_{2}$ atmosphere. Next, bacteria not internalized were removed by 10 successive vigorous washes with PBS at room temperature.

The macrophages were then placed back at $37^{\circ} \mathrm{C}$ to allow maturation to proceed for the indicated time. Where indicated, the cells were loaded with calcein blue by incubation with the precursor acetoxymethyl ester $(1 \mu \mathrm{g} / \mathrm{ml})$ for $10 \mathrm{~min}$. Internalized bacteria were identified by the area of calcein blue exclusion, while external adherent bacteria were discounted based on their position with respect to the blue-stained cytoplasm.

\section{Organellar Labeling}

To label lysosomal compartments, RAW macrophages were pulsed with $50 \mu \mathrm{g} / \mathrm{ml}$ of tetramethylrhodamine- or Alexa 647conjugated dextran $(10,000 \mathrm{MW})$ for $16 \mathrm{~h}$. The dextran-containing medium was then removed and the samples were chased in dye-free medium for $1 \mathrm{~h}$ to ensure that all contents were delivered to lysosomes. To label surface membranes, RAW macrophages were bathed in cold medium containing $1 \mu \mathrm{M}$ FM1-43 immediately before imaging, to prevent endocytosis. To detect unesterified cholesterol, RAW macrophages were fixed with $4 \%$ (vol/vol) paraformaldehyde in PBS for $30 \mathrm{~min}$, then quenched with $100 \mathrm{mM}$ glycine for $10 \mathrm{~min}$. Samples were then incubated with $50 \mu \mathrm{g} / \mathrm{ml}$ filipin overnight in the cold, washed several times with PBS, and then imaged.

\section{Electron Microscopy}

RAW macrophages were fixed in $2 \%$ glutaraldehyde in $0.2 \mathrm{M}$ Hepes, $\mathrm{pH} 7.4$, for $2 \mathrm{~h}$. After postfixation in 1\% osmium tetroxide, the cells were stained with $2 \%$ uranyl acetate, dehydrated in ethanol and flat-embedded in Epon. Thin sections were stained with uranyl acetate and lead citrate and examined with a Jeol JEM 1200 EX electron microscope.

Fluorescence Microscopy and Image Acquisition and Analysis Imaging of filipin-stained samples was performed using a Leica DMIRE2 microscope and Hamamatsu ORCA-ER camera. Openlab 4.0.4 software was used to drive image acquisition. All other fluorescence microscopy was performed using a Quorum spinning-disc microscopy system with a Zeiss Axiovert 200M microscope and either a Hamamatsu EM-CCD digital camera or a Hamamatsu ORCA 3CCD digital camera. Image acquisition, processing and analysis were driven by the Volocity 4.0 .0 software.

\section{Fluorescence Recovery after Photobleaching}

Cells grown on glass coverslips were transfected with either GFP-Rab7 or GFP-Rab7(Q67L) cDNA. After $24 \mathrm{~h}$, cells on coverslips were allowed to ingest either live or fixed bacteria for the indicated times, then transferred to Attofluor chambers that were mounted onto the heated stage of a laser scanning confocal microscope (Zeiss 510). Two baseline fluorescence images were acquired of at least $2 \mathrm{BcCVs}$ within the same cell. One selected $\mathrm{BcCV}$ was irreversibly photobleached and the fluorescence of both $\mathrm{BcCVs}$ was monitored at regular intervals over the indicated period. The fractional fluorescence recovery was calculated as a fraction of the average of the 2 prebleach measurements of the corresponding $\mathrm{BcCV}$. The unbleached $\mathrm{BcCV}$ was used to correct for potential bleaching incurred during the course of image acquisition.

\section{Statistics}

All experiments were performed at least in triplicate. Unless indicated otherwise, all data are presented as means \pm SE of the indicated number of determinations. For comparisons of means, paired t tests or analysis of variance were used, as appropriate. A probability of $<0.05$ was deemed significant. A signal was considered negative if the marker was absent from the vicinity of the phagosome or if its distribution around the phagosome was discontinuous, indicative of vesicle apposition without fusion.

\section{Results}

Opsonization of B. cenocepacia with Diluted Human Serum Enhances Phagocytosis

Experiments to pinpoint the stage of the maturation process affected by $B$. cenocepacia required synchronization of phagocy tosis. However, because these bacteria are not readily engulfed by macrophages, previous studies required extended coincubations to attain significant levels of internalization [7]. The asynchrony introduced by such long incubations is incompatible with accurate determination of the kinetics of maturation. To better synchronize phagocytosis and the subsequent maturation process, we sought means of shortening the period of exposure of the macrophages to $B$. cenocepacia. We incubated $\mathrm{J} 2315$ bacteria with diluted human serum, anticipating that they would be coated with complement proteins and possibly also IgG, which are ligands for phagocytic receptors of macrophages. Opsonized bacteria were sedimented onto macrophages to promote synchronous phagocytosis, and after $20 \mathrm{~min}$, external bacteria were removed by repeated, vigorous washes (see Materials and Methods). Antibiotics were not used because, due to the high resistance of $B$. cenocepacia to antimicro- 

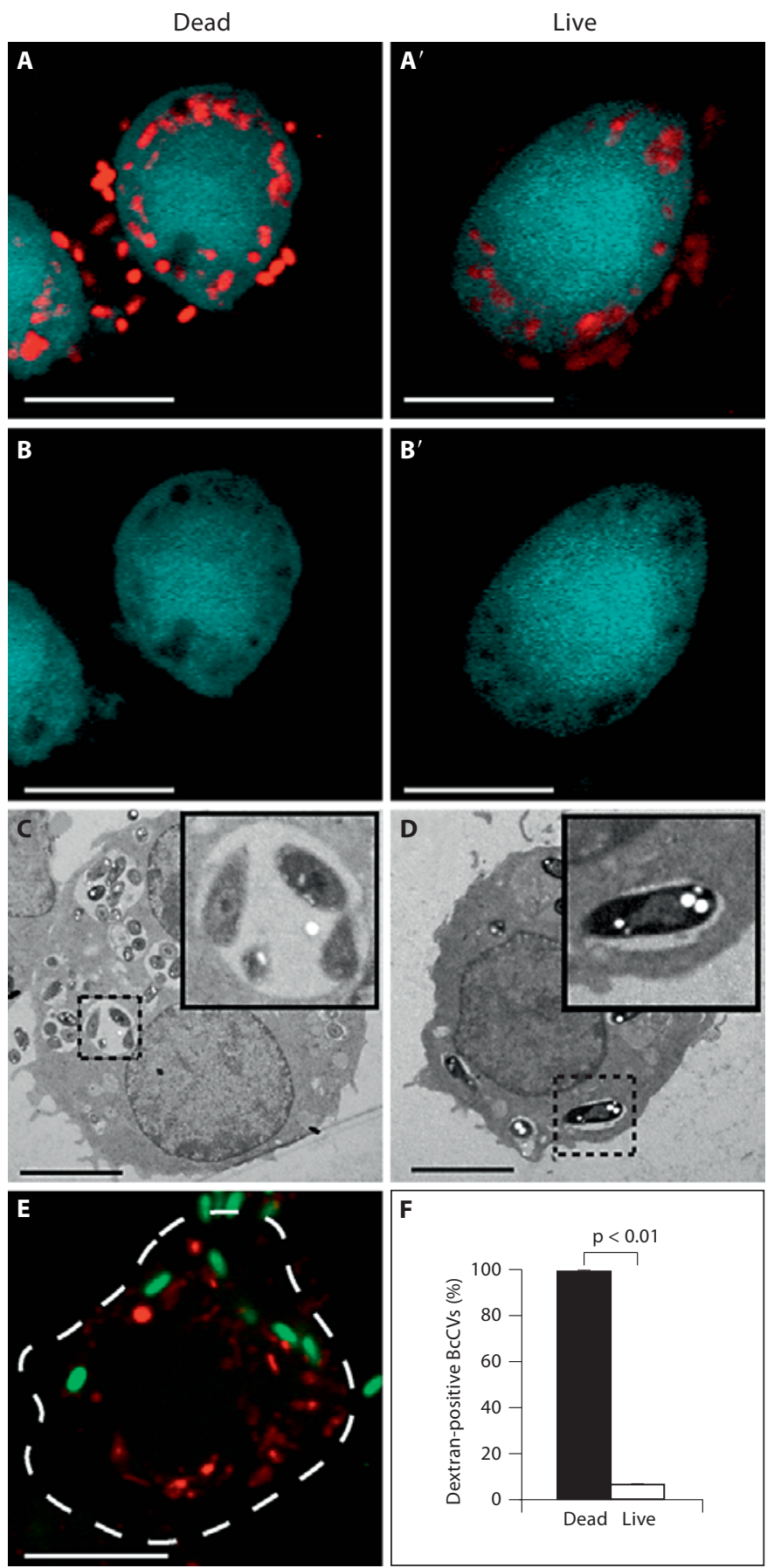

Fig. 1. Internalization of opsonized B. cenocepacia by macrophages. A, B RAW macrophages were labeled with calcein blue and then exposed for $20 \mathrm{~min}$ to either fixed (dead; A, B) or live $\left(\mathbf{A}^{\prime}\right.$, $\left.\mathbf{B}^{\prime}\right)$ B. cenocepacia expressing RFP, at a MOI of 60 bacteria/cell. The blue cytosolic dye was used to outline the cells and better differentiate intracellular bacteria, which generate a void in the blue outline (B, $\left.\mathbf{B}^{\prime}\right)$, from those adhering extracellularly. Washing was minimized in these experiments to illustrate extracellular bacteria. Scale bars $=10 \mu \mathrm{m}$. C, D Transmission electron micrographs of macrophages that were infected with either dead (C) or live (D) bial agents, especially aminoglycosides, addition of gentamicin or kanamycin to the growth medium fails to eliminate all extracellular bacteria [16]. Phagocytic efficiency was then determined by acquiring serial optical sections with a spinning disc confocal microscope, using the vital dye calcein blue to delineate the macrophage cytosol and thereby establish the intracellular location of engulfed bacteria. This dye was chosen for its spectral compatibility with either GFP- or RFP-expressing bacteria. As illustrated in figure $1 \mathrm{~A}, \mathrm{~B}$ (and in the online suppl. fig. 1, www.karger.com/doi/10.1159/000319864), internalized bacteria generated a void of calcein blue in the cytoplasm, caused by exclusion of the dye from the lumen of the $\mathrm{BcCV}$. That these bacteria were inside was validated using the impermeant solvatochromic dye FM1-43 (online suppl. fig. 1). Only extracellular adherent bacteria, but not those associated with voids in the calcein blue pattern, stained with the dye. Using this approach, when macrophages were exposed to live $B$. cenocepacia at a MOI of 60 for $20 \mathrm{~min}$, we found that $65.7 \pm 6 \%$ (mean \pm SE of 5 experiments) of the cells contained bacteria. On average, each cell contained $4.2 \pm 0.9$ bacteria. This is markedly higher than the index estimated for unopsonized B. cenocepacia after comparable or even longer times [7].

The improved engulfment efficiency obtained using serum-treated bacteria enabled better synchronization of phagocytosis, facilitating the study of maturation. However, it was important to determine whether by altering the path of internalization, opsonization affected the ability of the bacteria to arrest phagosome maturation. The fate of serum-opsonized live and heat-killed bacteria, used as a control, was compared. Similar to earlier results using unopsonized B. cenocepacia, BcCVs containing dead bacteria acquired lysosomal markers and within 60 min became highly acidic, as indicated by the accumulation of the acidotropic dye LysoTracker (not shown). In-

B. cenocepacia using the protocol described above. Insets are enlarged sections of the area indicated by the dotted line. Scale bars $=5 \mu \mathrm{m}$. See online supplementary figure 2 for additional examples. E Lysosomes were preloaded with tetramethylrhodamine-dextran (red), followed by infection with live, GFP-expressing $B$. cenocepacia (green) for $20 \mathrm{~min}$ followed by $60 \mathrm{~min}$ of maturation. F Quantitation of the fraction of dextran-positive BcCVs generated by dead or live bacteria in experiments like that illustrated in $\mathbf{E}$. Data are means \pm SE of 4 similar experiments, each counting at least 10 cells. 


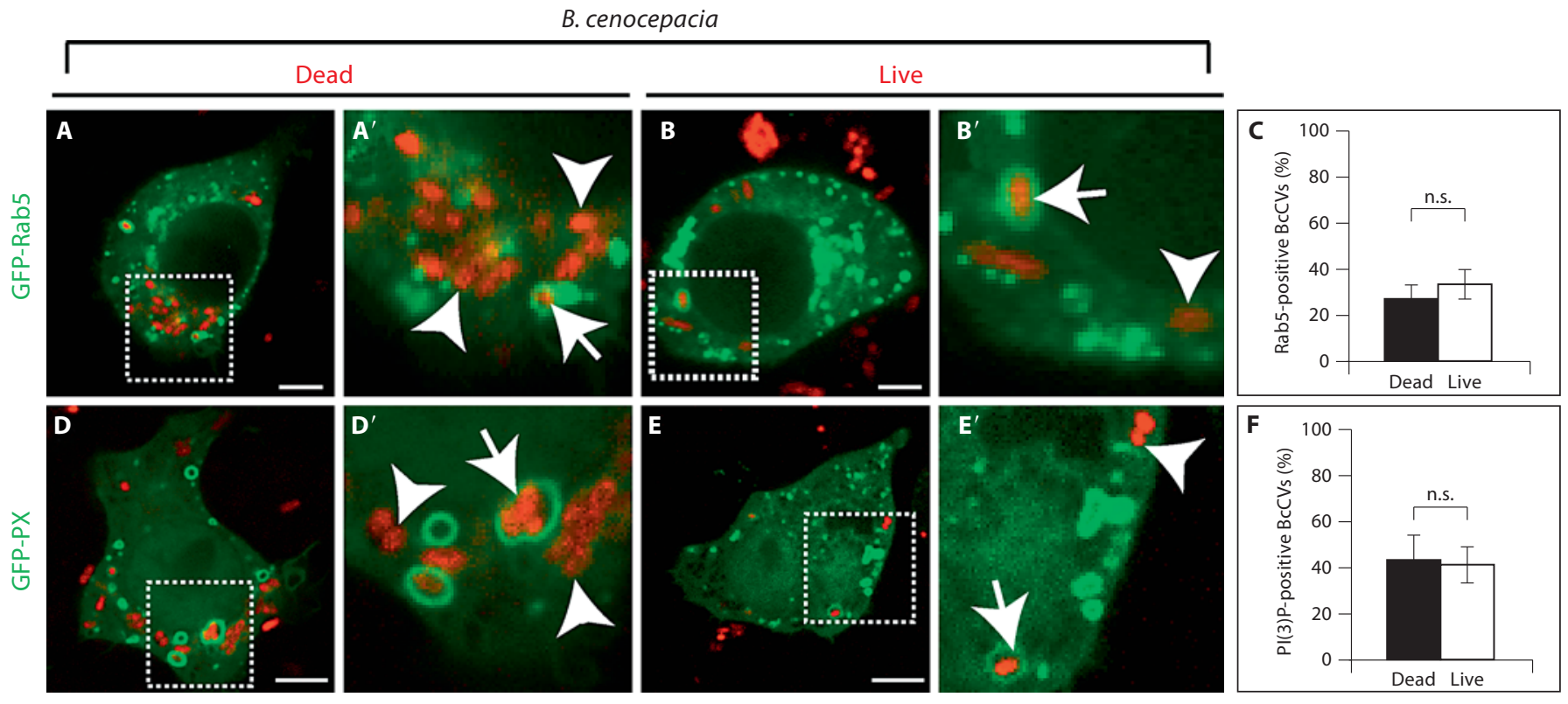

Fig. 2. Fusion of early endosomes with BcCVs. Macrophages were transfected with cDNA encoding either $\operatorname{Rab} 5(\mathbf{A}, \mathbf{B})$ or the PX domain of $\mathrm{p} 40^{\text {phox }}(\mathbf{D}, \mathbf{E})$ tagged with GFP, then infected with either dead $\left(\mathbf{A}, \mathbf{A}^{\prime}, \mathbf{D}, \mathbf{D}^{\prime}\right)$ or live (B, $\left.\mathbf{B}^{\prime}, \mathbf{E}, \mathbf{E}^{\prime}\right)$ B. cenocepacia for $20 \mathrm{~min}$. The bacteria, which expressed RFP, are shown in red. Enlargements of the areas demarcated by the dotted lines are shown in $\mathbf{A}^{\prime}, \mathbf{B}^{\prime}, \mathbf{D}^{\prime}$ and $\mathbf{E}^{\prime}$. Arrows indicate Rab5- or PX-domain-positive phagosomes, while arrowheads indicate negative phagosomes. Scale bars $=5 \mu \mathrm{m}$. The fraction of Rab5 (C) or PX-domain-positive (F) BcCVs containing dead (black bars) or live bacteria (white bars) was quantified in 3 similar experiments, each counting at least 10 cells. Data are means \pm SE. The differences between $\mathrm{BcCV}$ containing live and dead bacteria in $\mathbf{C}$ and $\mathbf{F}$ are not statistically significant (n.s.). deed, even 20 min after phagocytosis, the heat-killed bacteria were extensively degraded, often making their detection difficult. To improve preservation and ensure more accurate detection, bacteria were killed by fixation with $8 \%$ paraformaldehyde in all subsequent experiments. BcCVs containing fixed B. cenocepacia also accumulated LysoTracker rapidly (not shown). However, in contrast to heat-killed bacteria, the fixed bacteria were much better preserved at the time of analysis, improving detection. That the acidification was caused by fusion with lysosomes was confirmed using fluorescent dextrans. When loaded by a suitable pulse-chase protocol (see Materials and Methods), fluid-phase markers such as dextrans can label lysosomes unambiguously. As reported for unopsonized heat-killed bacteria, the vast majority (98.9 $\pm 0.4 \%$; mean $\pm \mathrm{SE}$ of 4 independent experiments) of the $\mathrm{BcCVs}$ containing serum-coated fixed bacteria were stained by lysosomal-targeted dextran $60 \mathrm{~min}$ after ingestion (fig. 1F).

We next analyzed the behavior of serum-exposed live B. cenocepacia. Like their unopsonized counterparts, a sizable fraction of the serum-coated bacteria eluded fusion with lysosomes (fig. 1E). After $60 \mathrm{~min}$, only $6.2 \pm$
$2 \%(n=4)$ of the live bacteria resided within dextranpositive BcCVs (fig. 1F). Failure to fuse with lysosomes was also noted when using lower MOI (online suppl. fig. 4), although quantitation was less accurate due to the lower number of vacuoles formed. Thus, the phenotype described earlier for unopsonized B. cenocepacia [7] was recapitulated by serum-coated bacteria. Because the latter were ingested much more efficiently, they were used for all subsequent studies, in conjunction with similarly coated bacteria previously killed by fixation, used as a control.

\section{Live B. cenocepacia Reside within a \\ Membrane-Bound Compartment}

Failure to fuse with lysosomes and to acidify could be caused by interference with the maturation sequence of the $\mathrm{BcCV}$, or by escape of the bacteria from the vacuole. The possibility that live $B$. cenocepacia can rupture the vacuole to reside in the cytosol was analyzed by electron microscopy. As illustrated in figure $1 \mathrm{D}$ and in online supplementary figure 2 , after live bacteria were internalized, they were routinely found in membrane-bound vacuoles, as were the fixed ones (fig. 1C). It is noteworthy that mul- 

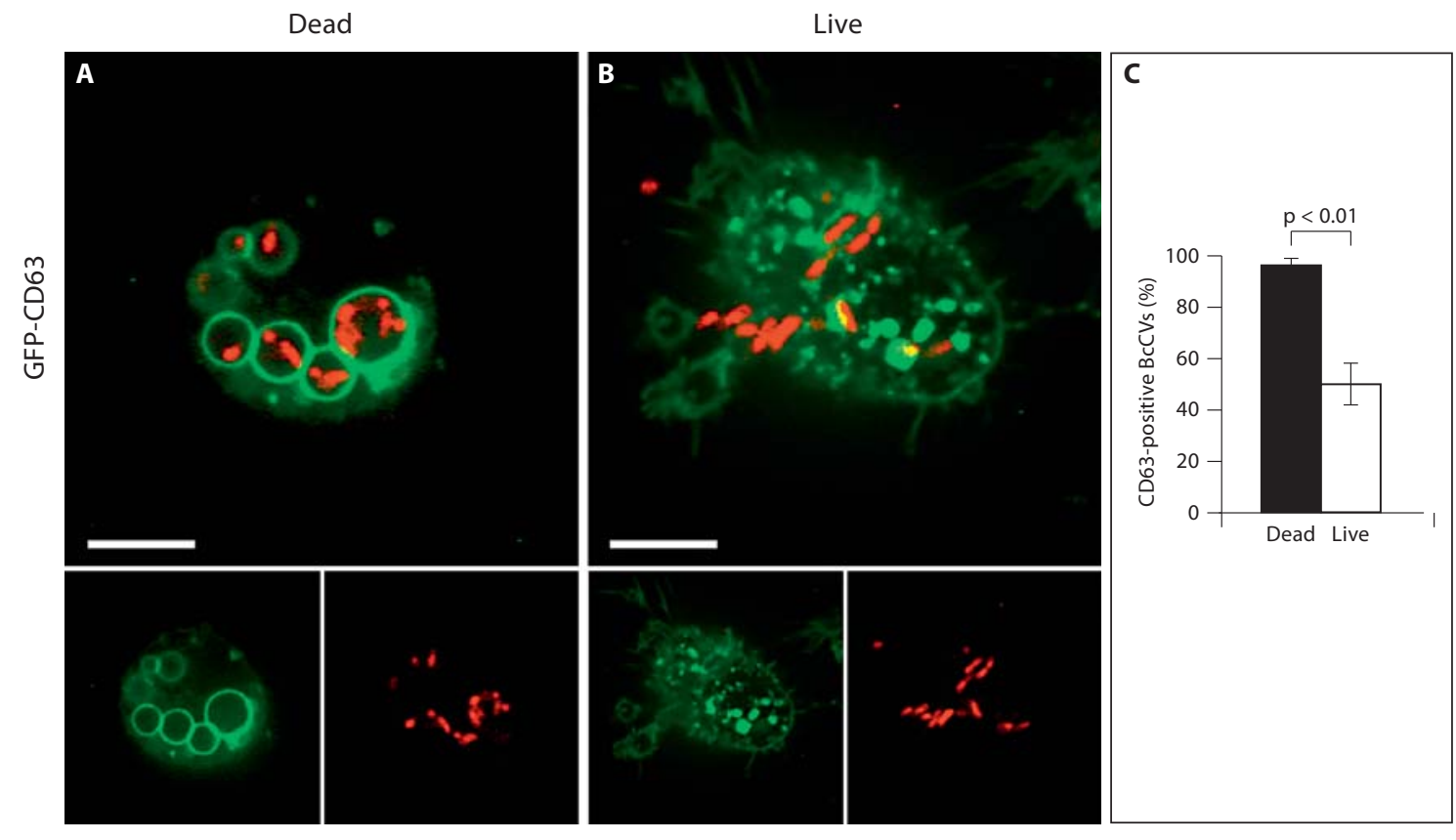

Fig. 3. Fusion of late endosomes/lysosomes with BcCVs. A, B Macrophages were transfected with cDNA encoding GFP-tagged CD63 then infected with either dead (A) or live (B) B. cenocepacia expressing RFP (red) for $20 \mathrm{~min}$ followed by $100 \mathrm{~min}$ of maturation. Scale bars $=5 \mu \mathrm{m}$. Lower panels in $\mathbf{A}$ and $\mathbf{B}$ show the red and

green channels separately. $\mathbf{C}$ The fraction of CD63-positive BcCVs containing dead (black bars) or live bacteria (white bars) was quantified in 5 similar experiments, each counting at least 10 cells. Data are means $\pm \mathrm{SE}$. The difference between BcCVs containing live and dead bacteria in $\mathbf{C}$ is highly significant $(\mathrm{p}<0.01)$.

tiple fixed bacteria were more frequently found in a single vacuole, compared with live ones. Because we ensured that the dead bacteria were well dispersed as singlets after fixation, this is suggestive of formation of compound vacuoles through fusion, a process that occurred rarely in the case of live B. cenocepacia.

\section{BcCVs Fuse with Early Endosomes}

Having validated the experimental model and recapitulated the failure of $\mathrm{BcCVs}$ to merge with lysosomes, we proceeded to study the stage and mechanism of maturation arrest. The earliest stage of phagosome maturation involves fusion with early endosomes and acquisition of Rab5. Macrophages were transfected with GFP-Rab5 to analyze whether this guanosine triphosphatase (GTPase) associates with BcCVs 20 min after ingestion (fig. 2A, B). Because the association with Rab5 is rapid and transient and because phagocytosis is not perfectly synchronous, only a fraction of the $\mathrm{BcCVs}$ was Rab5 positive at the time of analysis. Notably, the fraction of BcCVs associated with Rab5 was indistinguishable between live and dead bacteria (33.4 \pm 6.4 vs. $27.0 \pm 6.2 \%$, respectively; fig. $2 \mathrm{C}$ ), suggesting that $B$. cenocepacia does not interfere with the

earliest stages of the maturation process. This conclusion was supported by measurements of phosphatidylinositol3 -phosphate $[\mathrm{PI}(3) \mathrm{P}]$, which is formed on early phagosomes by the class III phosphatidylinositol-3-kinase, Vps34. The formation of PI(3)P was assessed by expression of a biosensor consisting of the PX domain of the p40 subunit of the NADPH oxidase conjugated to GFP [9]. As reported for phagosomes containing inert particles, $\mathrm{PI}(3) \mathrm{P}$ was found to accumulate transiently in early $\mathrm{BcCVs}$ (online suppl. fig. 3) and, as in the case of Rab5, only a fraction of the vacuoles was positive for the phosphoinositide at the time of analysis (fig. 2D, E). More importantly, the fraction of the BcCVs stained by the PI(3)P biosensor was similar for vacuoles containing live or dead bacteria ( $42.8 \pm 7.3$ vs. $43.5 \pm 10.9$, respectively; fig. 2F). Therefore, we found no evidence that B. cenocepacia interferes with the fusion of early endosomes with the BcCVs.

\section{Fusion of BcCVs with Late Endosomes/Lysosomes}

We next proceeded to study whether the $\mathrm{BcCVs}$ merge with late endosomes. At present, there are no effective markers that unambiguously distinguish late endosomes 

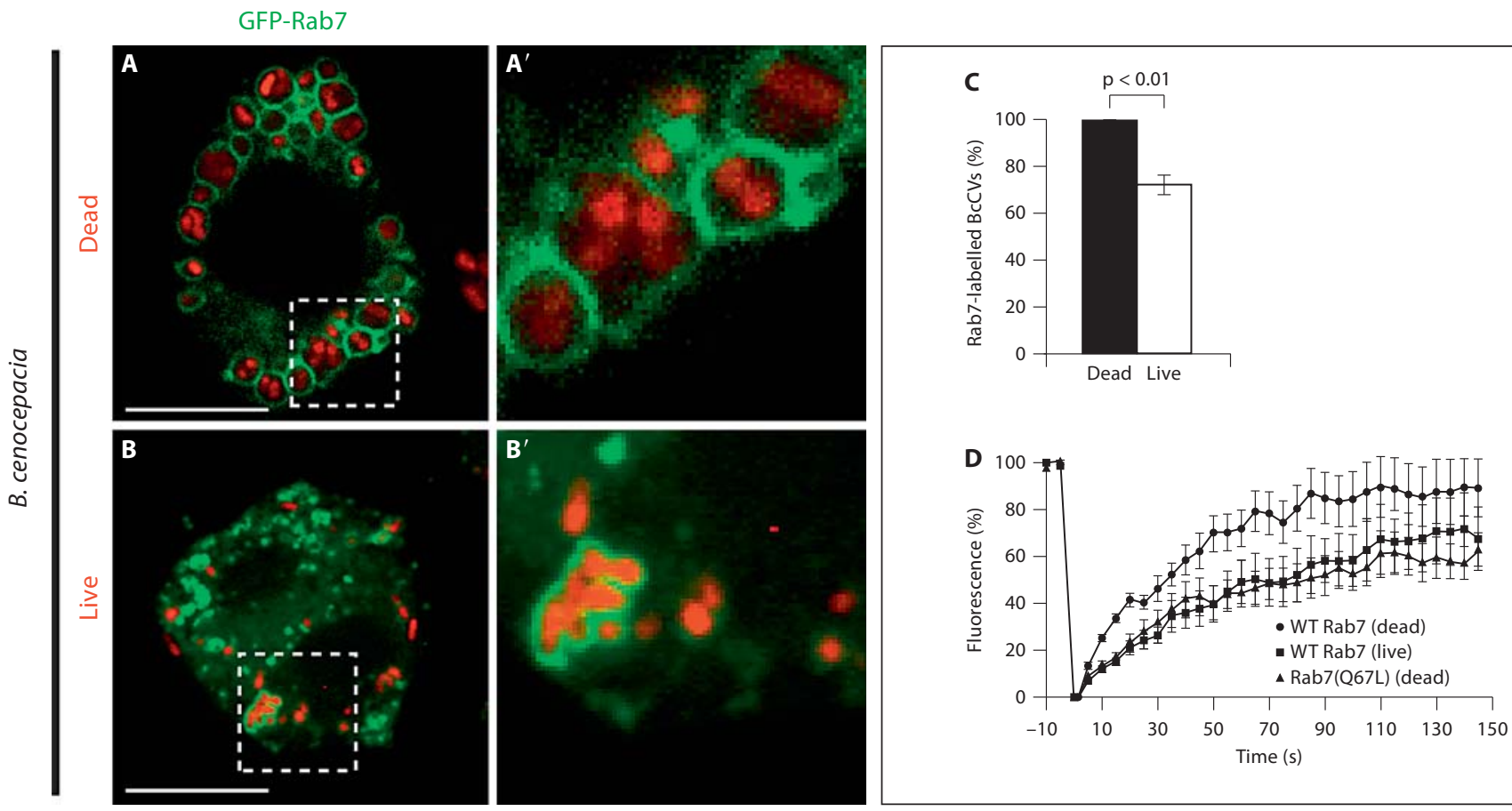

Fig. 4. Analysis of Rab7 in BcCVs. Macrophages were transfected with cDNA encoding GFP-tagged wild-type $\operatorname{Rab7}(\mathbf{A}, \mathbf{B})$, then infected with either fixed (A) or live (B) B. cenocepacia expressing RFP for $20 \mathrm{~min}$, followed by $100 \mathrm{~min}$ of maturation. $\mathbf{A}^{\prime}, \mathbf{B}^{\prime}$ Enlargements of the areas demarcated by the dotted lines. Scale bars = $5 \mu \mathrm{m}$. C The fraction of Rab7-positive BcCVs containing dead (black bars) or live bacteria (white bars) was quantified in 6 similar experiments, each counting at least 5 cells. Data are means \pm
SE. The difference between $\mathrm{BcCVs}$ containing live and dead bacteria in $\mathbf{C}$ is highly significant ( $\mathrm{p}<0.01)$. D FRAP of GFP-Rab7 on BcCVs. Macrophages were transfected with cDNA encoding GFPtagged wild-type Rab7 (WT) or constitutively active Rab7 [Rab7(Q67L)]. Cells were infected with either dead (fixed) or live bacteria as above. BcCVs were photobleached and the rate of fluorescence recovery monitored every $5 \mathrm{~s}$ for $145 \mathrm{~s}$. Data are means \pm SE of 4 separate determinations. from lysosomes, but a number of proteins are present in both compartments. One such marker is CD63, a tetraspanin protein found in the internal vesicles of multivesicular bodies, as well as in their limiting membrane and that of lysosomes $[17,18]$. To analyze the acquisition of $\mathrm{CD} 63$ by the BcCVs, opsonized bacteria were sedimented onto macrophages to promote synchronous phagocytosis and, after $20 \mathrm{~min}$, external bacteria were washed, and the formed vacuoles were allowed to mature for a further $100 \mathrm{~min}$. Because adequate antibodies for murine CD63 are not currently available, macrophages were transfected with cDNA encoding GFP-tagged CD63 (fig. 3A, B). As expected for inert particles, $96 \pm 2.7 \%$ $(n=5)$ of the $\mathrm{BcCVs}$ containing fixed bacteria were intensely labeled by GFP-CD63. In contrast to the BcCVs containing fixed B. cenocepacia, only $50 \pm 8.2 \%(n=5)$ of those containing live bacteria displayed GFP fluorescence (fig. 3B, C). While lower than the fraction containing dead bacteria, the percentage of $\mathrm{BcCV}$ s with live bac- teria that acquired CD63 was considerably higher than those fusing with dextran-labeled lysosomes $(50 \pm 8.2$ vs. $6.2 \%$, respectively). We interpret these results to mean that at least a fraction of the $\mathrm{BcCVs}$ merged with late endosomes.

\section{Acquisition and Activation of Rab7 by the BcCVs}

Conversion of an early to a late phagosome is marked by the transition between Rab5 and Rab7. If late endosomes are indeed fusing with BcCVs, Rab7 should be present on at least a fraction of the vacuoles. Because, as in the case of Rab5, reagents for detection of endogenous Rab7 are inadequate, macrophages were transfected with GFP-Rab7 prior to infection with $B$. cenocepacia. The transfected macrophages were allowed to ingest opsonized bacteria for $20 \mathrm{~min}$, followed by a 100 -min maturation period before analysis by spinning disc confocal microscopy (fig. 4). The vast majority $(99.3 \pm 0.5 \%$; $\mathrm{n}=$ 10) of the vacuoles containing dead bacteria acquired 
Rab7 during this period, while $71.9 \pm 4.2 \%(\mathrm{n}=10)$ of the $\mathrm{BcCVs}$ formed by live bacteria were Rab7 positive (fig. 4C). These findings support the notion that most $\mathrm{BcCV}$ s containing live bacteria can interact with late endosomes.

Rab7 is thought to direct the fusion of phagosomes not only with late endosomes, but also with lysosomes [19]. It was therefore unexpected to find that while most of the BcCVs formed by live B. cenocepacia acquire Rab7 (fig. $4 \mathrm{C}$ ), only a minute fraction of these proceed to merge with lysosomes. It was conceivable that, though recruited to the BcCVs, Rab7 was functionally inactive in the case of live bacteria. Fluorescence recovery after photobleaching (FRAP) of GFP-Rab7 was used to test this hypothesis. In the steady state, the rate of recovery of fluorescence following bleaching of the entire $\mathrm{BcCV}$ is dictated by exchange of the photo-bleached membrane-associated Rab7 with fluorescent (unbleached) cytosolic Rab7, a process that is determined in part by the rate of conversion of Rab7-guanosine diphosphate (GDP) to the GTP-bound form [for details, see ref. 16, 20]. Therefore, the rate of fluorescence recovery of Rab7 on vacuoles provides an indication of its activation state. To execute FRAP, wildtype GFP-Rab7 was expressed in macrophages, then phagocytosis of either dead or live B. cenocepacia was induced for $20 \mathrm{~min}$, followed by a 100 -min maturation period. Entire BcCVs decorated by Rab7 were photobleached and the rate of recovery of fluorescence was monitored over time. As shown in figure $4 \mathrm{D}$, photobleached $\mathrm{BcCVs}$ containing dead bacteria recovered their fluorescence rapidly $\left(\mathrm{t}_{1 / 2}=29 \pm 4.4 \mathrm{~s}\right)$, nearly regaining their original fluorescence (maximal recovery $=89.8 \pm 12.5 \%$ after $145 \mathrm{~s})$. These observations indicate fast, ongoing conversion between the active (GTP-bound) and inactive (GDPbound) forms of Rab7. Accordingly, when cells were transfected with GFP-tagged Rab7Q67L and allowed to ingest dead bacteria, the rates of fluorescence recovery were noticeably slower (fig. 4D). This constitutively active form of Rab7 cannot convert to the GDP-bound form and cannot be effectively removed from the membrane by Rab-GDP dissociation inhibitor. Exchange of membraneassociated Rab7 is then limited to other processes, such as membrane fusion and fission.

We next measured the rate of dissociation/reassociation of wild-type Rab7 in BcCVs containing live bacteria (fig. 4D). Remarkably, the rate of fluorescence recovery was slower and less complete than for BcCVs with dead B. cenocepacia $\left(\mathrm{t}_{1 / 2}=45 \pm 2.8 \mathrm{~s}\right.$, regaining only $67.9 \pm$ $13.7 \%$ of the starting fluorescence after $145 \mathrm{~s}$ ), approaching the rate and extent recorded using Rab7Q67L. These

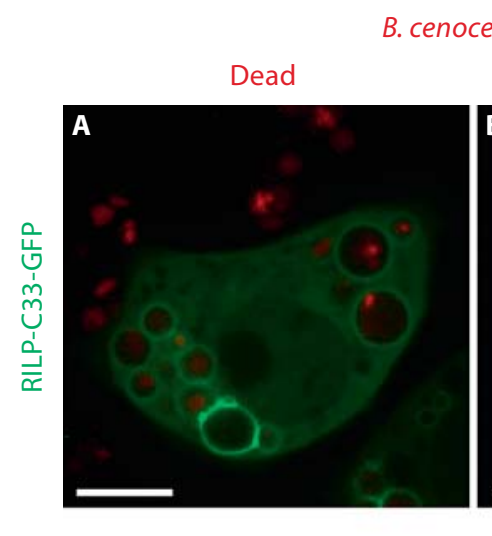

B. cenocepacia
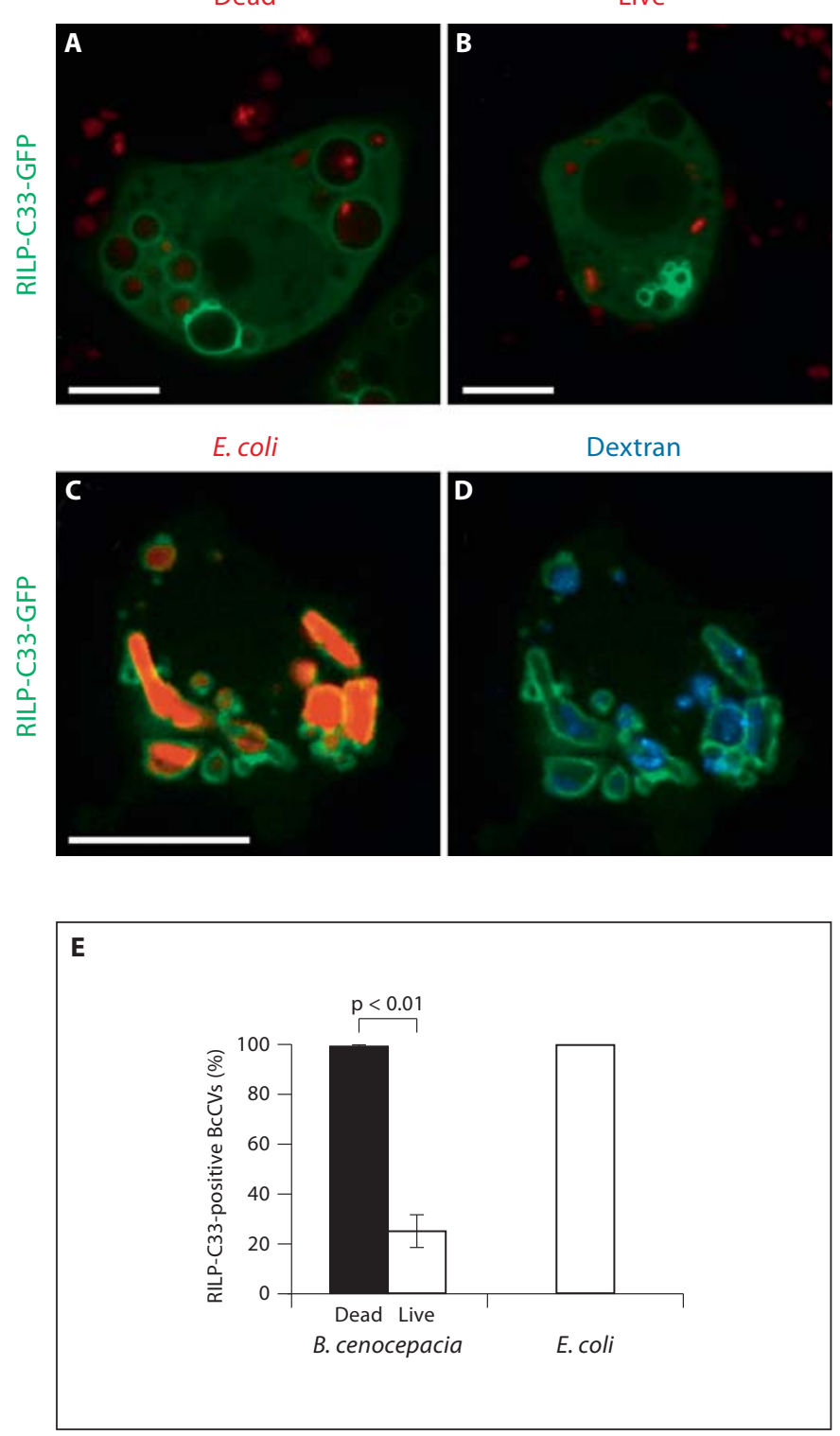

Fig. 5. Localization of active Rab7 in B. cenocepacia-infected macrophages. A, B Macrophages were transfected with cDNA encoding GFP-tagged RILP-C33, then infected with either fixed (A) or live (B) B. cenocepacia expressing RFP for $20 \mathrm{~min}$, followed by 100 min of maturation. C, D Macrophages were transfected with cDNA encoding GFP-tagged RILP-C33, and their lysosomes were loaded with Alexa 647-conjugated dextran (blue) as described in Materials and Methods. Next, the cells were infected with live $E$. coli expressing RFP for $20 \mathrm{~min}$, followed by $100 \mathrm{~min}$ of maturation. E The fraction of RILP-C33-GFP-positive BcCVs containing dead (black bars) or live bacteria (white bars) was quantified in 3 similar experiments, each counting at least 10 cells. The difference between $\mathrm{BcCVs}$ containing live and dead bacteria is highly significant $(\mathrm{p}<0.01)$. 
Fig. 6. Distribution of cholesterol in $B$. cenocepacia-infected macrophages. A-D Macrophages were infected with either fixed $\left(\mathbf{A}, \mathbf{B}, \mathbf{A}^{\prime}, \mathbf{B}^{\prime}\right)$ or live $\left(\mathbf{C}, \mathbf{D}, \mathbf{C}^{\prime}, \mathbf{D}^{\prime}\right)$ bacteria for $20 \mathrm{~min}$, followed by $100 \mathrm{~min}$ maturation, then fixed and stained with filipin. The distribution of filipin $\left(\mathbf{A}, \mathbf{A}^{\prime}, \mathbf{C}, \mathbf{C}^{\prime}\right)$ and the location of the RFP-expressing bacteria (B, B', D, $\left.\mathbf{D}^{\prime}\right)$ are shown separately. The outlines of the cells were traced manually in $\mathbf{B}$ and $\mathbf{D}$, for reference. $\mathbf{A}^{\prime}-\mathbf{D}^{\prime}$ Enlargements of the areas demarcated by the dotted lines in the main panels. Scale bars $=5 \mu \mathrm{m}$.
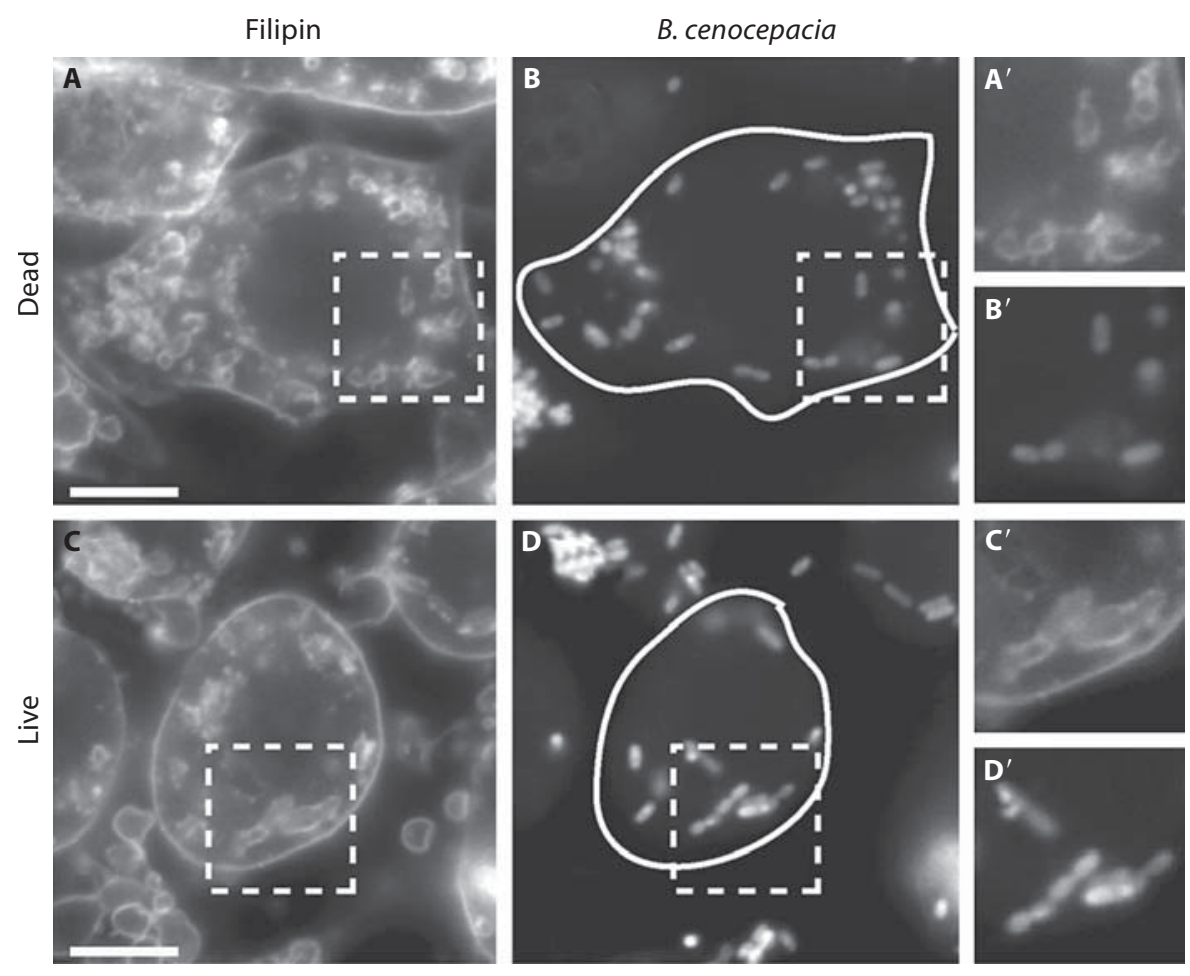

observations imply that the live bacteria affect the state of activation of Rab7 on the vacuole.

The preceding observations point to abnormal cycling between the GTP- and GDP-bound forms of Rab7 on $\mathrm{BcCVs}$ containing live bacteria, but cannot differentiate between sustained activation of the GTPase, or the reduced ability to extract from the membrane the inactive, GDP-bound form. To discern between these 2 possibilities, the activation state of Rab7 was analyzed by another approach. We took advantage of RILP, a Rab7 effector protein that exclusively recognizes the active (GTPbound) conformation of the GTPase [21]. When fused to GFP, the C-terminal, Rab7-binding domain of RILP called 'RILP-C33' can be used as a reliable index of the presence and distribution of active Rab7 [11]. As shown in figure 5A, E, RILP-C33-GFP strongly labels the vast majority $(99.3 \pm 0.5 ; \mathrm{n}=3)$ of $\mathrm{BcCVs}$ with dead bacteria. By contrast, only $25 \pm 6.6(n=3)$ of the vacuoles containing live B. cenocepacia were labeled with RILP-C33 (fig. 5B, E). Together, these observations indicate that the live bacteria inactivate and retain Rab7 on the vacuole, impairing fusion with lysosomes.

It is noteworthy that this crucial aspect of the phenotype is an inherent property of live B. cenocepacia and is not attributable to either the opsonization or infection protocol used for our experiments. Several lines of evidence support this notion. First, live nonpathogenic $E$. coli acquire RILP-C33 and, as expected, fuse with dextran-loaded lysosomes (fig. 5C-E; online suppl. fig. 4) at comparable MOI. Second, like the failure to acquire lysosomal dextran, the impairment in RILP-C33 recruitment was also noted when using lower MOI (online suppl. fig. 4). Lastly, defective RILP-C33 recruitment was also noted in live bacteria opsonized with heat-inactivated serum (online suppl. fig. 5).

\section{Involvement of Cholesterol in BcCV Maturation}

Previous research has demonstrated that accumulation of cholesterol in late endosomes/lysosomes alters the function of Rab GTPases, retaining the inactive GDPbound form in the membrane [22-25]. Moreover, excess cholesterol in late endosomes impairs Rab7 activation and inhibits phagosome maturation [26]. Since Rab7 was found to be similarly inactivated and retained in the membrane by live $B$. cenocepacia, we assessed whether cholesterol accumulates in the BcCV. Free (unesterified) cholesterol content was visualized in infected macrophages by staining with filipin (fig. 6). As expected, cholesterol was abundant in the plasma membrane, as well as in endocytic organelles, including the BcCV. However, 
Fig. 7. Interaction of $\mathrm{BcCVs}$ containing live and dead bacteria with lysosomes. Lysosomes were preloaded with Alexa 647-conjugated dextran (blue), followed by infection with live, GFP-expressing B. cenocepacia (green) for $20 \mathrm{~min}$ and washing. Next, the cells were exposed to RFP-expressing dead B. cenocepacia (red) for an additional $20 \mathrm{~min}$, followed by washing and a final $40 \mathrm{~min}$ incubation to allow maturation. A representative image is shown in $\mathbf{A}$, and quantitation of 4 similar experiments is summarized in B. Scale bar $=5 \mu \mathrm{m}$. The difference between BcCVs containing live and dead bacteria is highly significant $(\mathrm{p}<0.01)$.
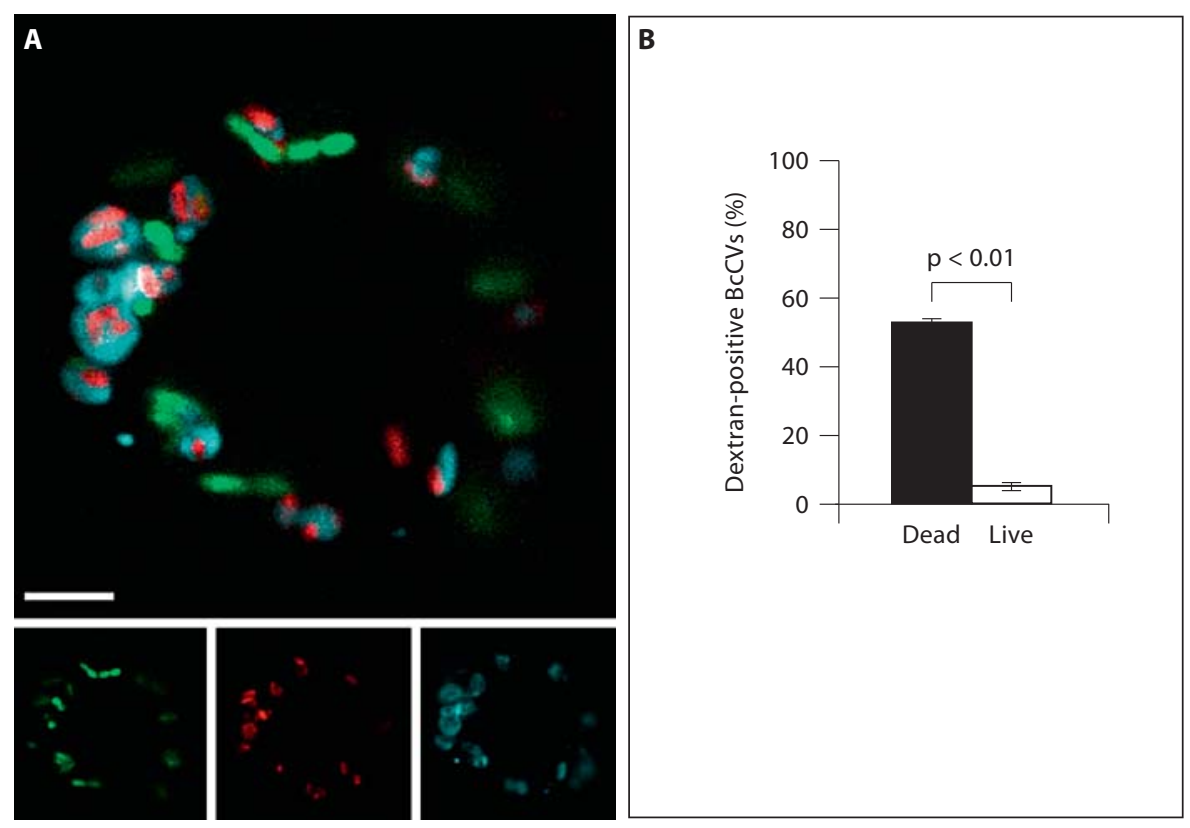

there was no discernible difference between the amounts of cholesterol in BcCVs containing live versus dead $B$. cenocepacia. These data suggest that live $B$. cenocepacia impaired Rab7 activation on phagosomes by a mechanism not involving excessive sequestration of cholesterol.

\section{Is Impairment of Lysosomal Fusion a Localized or \\ Generalized Phenomenon?}

The preceding data indicate that live $B$. cenocepacia impair the activation of Rab7 and thereby prevent fusion of the $\mathrm{BcCVs}$ with lysosomes. The bacteria may be selectively inhibiting Rab7 function on the vacuolar membrane, or may instead deliver an effector that inhibits Rab7 globally, throughout the cell. We used fluorescent dextran labeling to assess if live B. cenocepacia impair only the fusion of those $\mathrm{BcCVs}$ where they reside, or whether they prevent lysosomal fusion in general. Lysosomes were preloaded with Alexa 647-conjugated dextran, followed by infection with live, GFP-expressing $B$. cenocepacia for $20 \mathrm{~min}$ and washing. Next, the cells were exposed to RFP-expressing, fixed (dead) B. cenocepacia for an additional $20 \mathrm{~min}$, followed by washing and a final 40-min incubation to allow maturation. A representative experiment is illustrated in figure 7A. As before, $\mathrm{BcCVs}$ with live bacteria failed to fuse with lysosomes (only 5.4 $\pm 3.8 \%$ were dextran positive), while in the same cells, those containing dead bacteria fused efficiently (53.1 \pm $2.5 \%$ dextran positive), despite the shorter time they were allowed to mature (fig. 7B). These findings imply that the effects of live B. cenocepacia on fusion competence are restricted to the vacuole where they are contained and not disseminated throughout the cell.

\section{Discussion}

In agreement with earlier observations [7], we found that $B$. cenocepacia is taken up very poorly by macrophages. Whether the bacteria lack ligands recognized by the phagocytic receptors of leukocytes or exert an active antiphagocytic effect remains to be established. In this regard, it was recently shown that surface lipopolysaccharide affects the extent of phagocytosis of $B$. cenocepacia [27]. Regardless of the underlying mechanism, the low phagocytic efficiency complicates the analysis of the maturation of the $\mathrm{BcCV}$ for 2 reasons: first, the comparatively long duration required for ingestion of a sufficient number of bacteria compromises the definition of the kinetics of maturation. Secondly, because some of the probes required for the analysis must be transfected into the cells, the low probability of phagocytosis is compounded by the similarly low probability of transfection, usually in the range of $10 \%$ for RAW cells. We were able to overcome this limitation by incubating the cells in diluted human serum, which likely resulted in complement deposition on their surface, via the alternative pathway. 
The experimental system was also improved by using paraformaldehyde-fixed, as opposed to heat-killed, bacteria as controls. Fixation better preserved the bacteria, making the microscopic determinations more accurate. It is noteworthy that, unlike the tight vacuoles that contained live B. cenocepacia, the $\mathrm{BcCVs}$ formed around fixed bacteria were spacious, likely because they contained osmotically active debris released by partial digestion of the bacteria. This was suggested by the presence of grainy, modestly electron-dense material in micrographs like that shown in figure $1 \mathrm{C}$. Thus, fixation retarded, but did not completely prevent digestion of the dead bacteria by lysosomal enzymes.

Using this improved experimental model, we determined that Rab5 is recruited by the $\mathrm{BcCVs}$, which are also capable of synthesizing PI(3)P, implying acquisition of early phagosomal properties and likely the ability to fuse with early endosomes. This conclusion is in accordance with our earlier observation that the $\mathrm{BcCV}$ remains accessible to fluid phase markers taken up by endocytosis after infection [7]. Remarkably, despite the failure of most $\mathrm{BcCVs}$ to merge with lysosomes, they nevertheless acquired CD63 and Rab7, suggesting that they proceeded to the late phagosomal stage, equivalent to the late endosomal stage. Since the recruitment of Rab7 to the $\mathrm{BcCV}$ was not prevented, we speculated that Rab7 could be functionally impaired on the $\mathrm{BcCV}$. Two lines of evidence support this contention: (1) the abnormal rate of dissociation/reassociation of Rab7 from BcCVs, determined using FRAP; and (2) that despite the presence of Rab7, $\mathrm{BcCV}$ s were unable to recruit C33-RILP, a probe for the active form of the GTPase.

Because active Rab7 is required for phagolysosomal fusion [19], the observed impairment of Rab7-GTP formation could account for the arrested maturation of the $\mathrm{BcCV}$. To test this hypothesis, we assessed the fate of live B. cenocepacia in macrophages that had been transfected with the active mutant of Rab7 [Rab7(Q67L)] (online suppl. fig. 6). Despite successful expression and recruitment of the active form of Rab7 to the vacuole, fusion with lysosomes was not restored; the extent of dextran acquisition was similar to that seen in cells expressing the wildtype form of Rab7 (online suppl. fig. 6). We interpret this finding to mean that while inactivation of Rab7 may contribute to prevent fusion of lysosomes with the $\mathrm{BcCV}$, other mechanisms are also deployed, and that restoration of Rab7 activity alone is insufficient to rehabilitate maturation. It is also noteworthy that the inhibition of fusion may not be permanent, since fusion of the $\mathrm{BcCV}$ with the late endosomal/lysosomal marker LAMP (lysosomal-as- sociated membrane protein) was described to progress after a delay of about $6 \mathrm{~h}$ [7].

Since the BcCV appears to be an intact, sealed membrane compartment, judged by electron microscopy and by the exclusion of calcein blue, it is interesting to consider the mechanism whereby $B$. cenocepacia alters the function of Rab7, a protein localized on the contralateral (cytosolic) face of the vacuole. B. cenocepacia possess a type III secretion system that could readily account for the delivery of effectors to the cytosol. However, earlier observations indicated that the type III secretion system is dispensable for maturation arrest [7]. These bacteria also express type IV [28] and type VI [29] secretion mechanisms that may be responsible for the observed phenotype. The identity of the effectors secreted by these or other as yet unknown secretory pathways, their mode of action and the manner in which they reach the cytosol remain unclear. A better definition of the role of virulence factors interfering with the phagosomal maturation pathway will be required to better understand the remarkable ability of $B$. cenocepacia to escape killing and survive inside the host phagocytes.

\section{Acknowledgments}

This work was supported by grants from the Canadian Cystic Fibrosis Foundation (CCFF) and the Canadian Institutes of Health Research (CIHR; grant No. 7075) and the National Institutes of Health (HL090669 to G.P.D.). K.K.H. was the recipient of CCFF and CIHR Graduate Studentships. M.A.V. holds a Canada Research Chair in Infectious Diseases and Microbial Pathogenesis. S.G. is the current holder of the Pitblado Chair in Cell Biology.

References $\quad 1$ Goldman MJ, et al: Human beta-defensin-1 is a salt-sensitive antibiotic in lung that is inactivated in cystic fibrosis. Cell 1997;88:553560.

$\checkmark 2$ Matsui H, et al: Evidence for periciliary liquid layer depletion, not abnormal ion composition, in the pathogenesis of cystic fibrosis airways disease. Cell 1998;95:1005-1015.

-3 Smith JJ, et al: Cystic fibrosis airway epithelia fail to kill bacteria because of abnormal airway surface fluid. Cell 1996;85:229-236.

4 Waters V, Ratjen F: Multidrug-resistant organisms in cystic fibrosis: management and infection-control issues. Expert Rev Anti Infect Ther 2006;4:807-819.

5 Mahenthiralingam E, Urban TA, Goldberg JB: The multifarious, multireplicon Burkholderia cepacia complex. Nat Rev Microbiol 2005;3:144-156. 
6 Vanlaere E, et al: Burkholderia latens sp. nov., Burkholderia diffusa sp. nov., Burkholderia arboris sp. nov., Burkholderia seminalis sp. nov. and Burkholderia metallica sp. nov., novel species within the Burkholderia cepacia complex. Int J Syst Evol Microbiol 2008;58:1580-1590.

$\checkmark 7$ Lamothe J, et al: Intracellular survival of Burkholderia cenocepacia in macrophages is associated with a delay in the maturation of bacteria-containing vacuoles. Cell Microbiol 2007;9:40-53.

$\checkmark 8$ Saldías MS, Valvano MA: Interactions of Burkholderia cenocepacia and other Burkholderia cepacia complex bacteria with epithelial and phagocytic cells. Microbiology 2009;155:2809-2817.

-9 Kanai F, et al: The PX domains of p47phox and $\mathrm{p} 40$ phox bind to lipid products of PI(3) K. Nat Cell Biol 2001;3:675-678.

10 Roberts RL, et al: Endosome fusion in living cells overexpressing GFP-Rab5. J Cell Sci 1999; 112:3667-3675.

11 Cantalupo G, et al: Rab-interacting lysosomal protein (RILP): the Rab7 effector required for transport to lysosomes. EMBO J 2001;20: 683-693.

12 Bucci C, et al: Rab7: a key to lysosome biogenesis. Mol Biol Cell 2000;11:467-480.

13 Zuccato E, et al: Sorting of Fas ligand to secretory lysosomes is regulated by monoubiquitylation and phosphorylation. J Cell Sci 2006;120:191-199.
14 Johnson WM, Tyler SD, Rozee KR: Linkage analysis of geographic and clinical clusters in Pseudomonas cepacia infections by multilocus enzyme electrophoresis and ribotyping. J Clin Microbiol 1994;32:924-930.

15 Govan JR, et al: Evidence for transmission of Pseudomonas cepacia by social contact in cystic fibrosis. Lancet 1993;342:15-19.

16 Saini LS, et al: Intracellular survival of Burkholderia cepacia complex isolates in the presence of macrophage cell activation. Microbiology 1999; 145:3465-3475.

17 Kobayashi T, et al: The tetraspanin CD63/ lamp3 cycles between endocytic and secretory compartments in human endothelial cells. Mol Biol Cell 2000;11:1829-1843.

18 Escola JM, et al: Selective enrichment of tetraspan proteins on the internal vesicles of multivesicular endosomes and on exosomes secreted by human B-lymphocytes. J Biol Chem 1998;273:20121-20127.

19 Harrison RE, et al: Phagosomes fuse with late endosomes and/or lysosomes by extension of membrane protrusions along microtubules: role of Rab7 and RILP. Mol Cell Biol 2003;23:6494-6506.

20 Vieira OV, et al: Modulation of Rab5 and Rab7 recruitment to phagosomes by phosphatidylinositol 3-kinase. Mol Cell Biol 2003;23:2501-2514.

-21 Jordens I, et al: The Rab7 effector protein RILP controls lysosomal transport by inducing the recruitment of dynein-dynactin motors. Curr Biol 2001;11:1680-1685.
22 Lebrand C, et al: Late endosome motility depends on lipids via the small GTPase Rab7. EMBO J 2002;21:1289-1300.

23 Choudhury A, et al: Elevated endosomal cholesterol levels in Niemann-Pick cells inhibit Rab4 and perturb membrane recycling. Mol Biol Cell 2004;15:4500-4511.

24 Takahashi M, et al: Cholesterol controls lipid endocytosis through Rab11. Mol Biol Cell 2007; 18:2667-2677.

$>25$ Ganley IG, Pfeffer SR: Cholesterol accumulation sequesters Rab9 and disrupts late endosome function in NPC1-deficient cells. J Biol Chem 2006;281:17890-17899.

-26 Huynh KK, Gershenzon E, Grinstein S: Cholesterol accumulation by macrophages impairs phagosome maturation. J Biol Chem 2008;283:35745-35755

$\checkmark 27$ Saldías MS, Ortega X, Valvano MA: Burkholderia cenocepacia $\mathrm{O}$ antigen lipopolysaccharide prevents phagocytosis by macrophages and adhesion to epithelial cells. J Med Microbiol 2009;58:1542-1548.

28 Sajjan SU, et al: A type IV secretion system contributes to intracellular survival and replication of Burkholderia cenocepacia. Infect Immun 2008;76:5447-5455.

29 Aubert DF, Flannagan RS, Valvano MA: A novel sensor kinase-response regulator hybrid controls biofilm formation and type VI secretion system activity in Burkholderia cenocepacia. Infect Immun 2008;76:19791991. 